\title{
Salvador-Warts-Hippo pathway regulates sensory organ development via caspase-dependent nonapoptotic signaling
}

\author{
Lan-Hsin Wang ${ }^{1}$ and Nicholas E. Baker ${ }^{2,3,4}$
}

\begin{abstract}
The fundamental roles for the Salvador-Warts-Hippo (SWH) pathway are widely characterized in growth regulation and organ size control. However, the function of SWH pathway is less known in cell fate determination. Here we uncover a novel role of the SWH signaling pathway in determination of cell fate during neural precursor (sensory organ precursor, SOP) development. Inactivation of the SWH pathway in SOP of the wing imaginal discs affects caspase-dependent bristle patterning in an apoptosis-independent process. Such nonapoptotic functions of caspases have been implicated in inflammation, proliferation, cellular remodeling, and cell fate determination. Our data indicate an effect on the Wingless $(\mathrm{Wg})$ Wht pathway. Previously, caspases were proposed to cleave and activate a negative regulator of Wg/Wnt signaling, Shaggy (Sgg)/GSK3ß. Surprisingly, we found that a noncleavable form of Sgg encoded from the endogenous locus after CRISPR-Cas9 modification supported almost normal bristle patterning, indicating that Sgg might not be the main target of the caspase-dependent nonapoptotic process. Collectively, our results outline a new function of SWH signaling that crosstalks to caspase-dependent nonapoptotic signaling and Wg/Wnt signaling in neural precursor development, which might be implicated in neuronal pathogenesis.
\end{abstract}

\section{Introduction}

The Salvador-Warts-Hippo (SWH) pathway has been recognized as a significant regulator for growth control, tissue regeneration, and stem cell pluripotency ${ }^{1,2}$. It has also been found to play important roles in cancer metastasis $^{3-5}$. Originally identified as a prominent regulator of organ size in Drosophila, this pathway is highly conserved from fly to mammals. The regulation of SWH pathway depends on its response to various upstream stimuli through intercellular junctions, including adhesion cues

Correspondence: Lan-Hsin Wang (lanhsinwang.sinica@gmail.com) or Nicholas E. Baker (nicholas.baker@einstein.yu.edu)

'Graduate Institute of Life Sciences, National Defense Medical Center, $161 \mathrm{Sec}$ 6, Minquan E. Rd, Taipei 11490, Taiwan

${ }^{2}$ Department of Genetics, Albert Einstein College of Medicine, 1300 Morris Park Avenue, Bronx, NY 10461, USA

Full list of author information is available at the end of the article.

Edited by E. Baehrecke through cell-cell contact, polarity, extracellular signal, mechanical signals, and cellular stress. Core components of the SWH pathway comprise a kinase cascade, which is the main regulation modulating SWH signaling. The Ste20 family kinase Hpo (MST1/2 in mammals) forms a heterodimer with the adapter protein Sav (SAV or WW45 in mammals), thereby promoting their interaction with the serine/threonine kinase Wts (LATS1/2 in mammals). Hpo subsequently activates Wts activity via phosphorylation ${ }^{6,7}$. The activated Wts kinase operates with its cofactor Mats (MOB1A/B in mammals) to phosphorylate Yorkie (Yki; YAP and TAZ in vertebrates), which is a transcriptional coactivator and serves as the final effector of the Hippo signaling. Yki/YAP/TAZ lacks DNA-binding motif but binds to the promoters of target genes through interacting with Scalloped (Sd, TEAD1-4 in mammals) or other transcription factors ${ }^{6}$. By activating target gene expression, $\mathrm{Yki} / \mathrm{YAP} / \mathrm{TAZ}$ plays important roles in controlling cell growth, proliferation, and survival. Wts kinase inhibits

\section{(c) The Author(s) 2019}

(c) (i) Open Access This article is licensed under a Creative Commons Attribution 4.0 International License, which permits use, sharing, adaptation, distribution and reproduction c. in any medium or format, as long as you give appropriate credit to the original author(s) and the source, provide a link to the Creative Commons license, and indicate if changes were made. The images or other third party material in this article are included in the article's Creative Commons license, unless indicated otherwise in a credit line to the material. If material is not included in the article's Creative Commons license and your intended use is not permitted by statutory regulation or exceeds the permitted use, you will need to obtain permission directly from the copyright holder. To view a copy of this license, visit http://creativecommons.org/licenses/by/4.0/. 
transcriptional activity of Yki/YAP/TAZ through nuclear export, cytoplasmic retention, and protein degradation ${ }^{8-}$ ${ }^{10}$. Phosphorylation-independent regulations also exist. Yki can directly bind to Hpo, Wts and the FERM-domain containing adapter protein expanded (Ex), and sequesters Yki in the cytoplasm ${ }^{11,12}$. In addition to intrinsically regulates Yki activity, Ex, as an apical junctions-localized protein, also transduces signaling cues through binding to the apical membrane protein Crumbs (Crb, CRB3 in mammals $)^{13-16}$. In addition, $e x$ is a downstream target gene of Yki, thereby forming a feedback regulatory loop of Hippo pathway ${ }^{17}$. It has been demonstrated that activation of the SWH pathway through elevating expanded (ex) levels is required to eliminate the inappropriately differentiating neurons during development ${ }^{18,19}$. However, whether SWH pathway has any roles in normal neurogenesis remained unclear.

Intriguingly, hypomorphic ex mutants often differentiate supernumerary sensory bristles ${ }^{18}$. Bristles are a component of the Drosophila peripheral nervous system and can be divided into macro- (large bristles) and microchaetae (small bristles) according to their size and position. Drosophila notum is a classical model to study pattern formation because each macrochaetae develop in precise positions and microchaetae appears in a characteristic density pattern ${ }^{20}$. Each of these external sensory organs comprises five cells (hair, socket, neuron, sheath cell, and glial cell) that are generated through asymmetric cell divisions of single sensory organ precursor (SOP) cell $^{21,22}$. The accuracy of bristle patterns on the adult body depends on the correct SOP cell positioning. The phenotype of ex mutations promoted us to study in depth how $e x$ mediates sensory organ development.

Caspase activation has been implicated in SOP development through a caspase-dependent nonapoptotic machinery. This caspase-dependent machinery is thought to be required for cleavage and activation of a negative regulator of Wingless (Wg)/Wnt signaling, Shaggy (Sgg)/GSK3 $\beta$, in SOP cell formation ${ }^{23}$. By studying how ex takes part in SOP development, we discovered a crosstalk between SWH pathway and caspase-dependent nonapoptotic signaling mediated through Wg pathway. Interestingly and unexpectedly, we found Sgg might not be the main target of the caspase-dependent nonapoptotic event.

\section{Materials and methods}

\section{Mutants and transgenes}

$$
e x^{1}, e x^{e 1}, e^{69724} ; \text { Diap }^{125} ; \text { Diap }^{426} ; \text { dsh }^{327} ; \text { arm }^{2}, \text { arm }^{328}
$$

are loss-of-function or null alleles. Other transgenes used in this study include UAS-ex-RNAi (BDSC BL\#28703); UAS-yki, $U A S-y k i^{S 168 A}{ }^{10}$; UAS-dTCF[DN] (BDSC BL\#4784); UASdTCF-RNAi (BDSC BL\#26743); dpp ${ }^{40 C 6}-$ Gal $^{29} ; E^{\text {Intron3 }}$ $G F P^{18}$.

\section{Immunohistochemistry and histology}

Preparation of wing discs for immunostaining and adult notum for light microscope was performed as described previously ${ }^{30}$. Confocal imaging was performed using Leica SP2, SP8 and Zeiss LSM 880 microscopy. Primary antibodies used were anti-Sens (guinea pig, a gift from $\mathrm{H}$. Bellen); GFP (rat, NACALAI TESQUE\# GF090R). Photograph of adult notum was carried out using Leica MZFLIII microscope and Nikon SMZ1500 microscope.

\section{CRISPR/Cas9-based genome editing of sgg gene}

By using CRISPR/Cas9-mediated genome editing, mutagenesis of the corresponding genomic sequences in both 235th and 300th Asp residues of $s g g-\mathrm{RD} / \mathrm{RP} / \mathrm{RQ}$ isoforms were conducted in $w^{1118}$ flies. Two single guide RNAs (sgRNAs) were used to introduce double strand breaks near by the edited genomic region and followed by homology-directed repair (HDR). The HDR donor plasmid was designed to harbor a DNA cassette containing the upstream homology arm of $s g g$, 3XP3-ScarlessDsRed flanking with PiggyBac terminal repeats, and the downstream homology arm of $s g g$ with D235G/D300G mutations, which was constructed into the pUC57-Kan vector. The sgRNA and HDR donor plasmids used for microinjection were purified using the Plasmid Midi-prep kit (Qiagene). After validation of the CRISPR-knockin sgg alleles by genomic PCR coupled with Sanger sequencing, the ScarlessDsRed selection marker was then excised by PiggyBac transposon. The genomic PCR coupled with Sanger sequencing was performed to confirm the precise excision of ScarlessDsRed.

\section{Results}

\section{Ex is required to suppress extra macrochaete in the} scutellum

Reduced ex function in Drosophila by using transheterozygous ex mutants caused the appearance of ectopic macrochaete on the notum (Fig. 1b, c). Knockdown of $e x$ in the scutellum, using the $d p p$-GAL4 driver, also resulted in the formation of extra macrochaete in $62.5 \%$ of flies (Fig. 1d). Compared with normal macrochaete, the extra macrochaete observed in ex mutants were occasionally thinner and shorter, but still contained socket cells of normal morphology (Fig. 1d'). These hypomorphic ex genotypes survived to adulthood without obvious growth defects in the scutellum (Supplementary Fig. 1). To address whether the extra macrochaetae were produced from extra SOP cells, the SOP cells were visualized by Senseless (Sens) staining. Normally, two sets of SOPs (one anterior scutellar (aSC) and one posterior scutellar (pSC) bristles, respectively) exist on the scutellum of one wing imaginal disc, whereas more than two SOP cells were detected when ex was downregulated (Fig. 2). These 

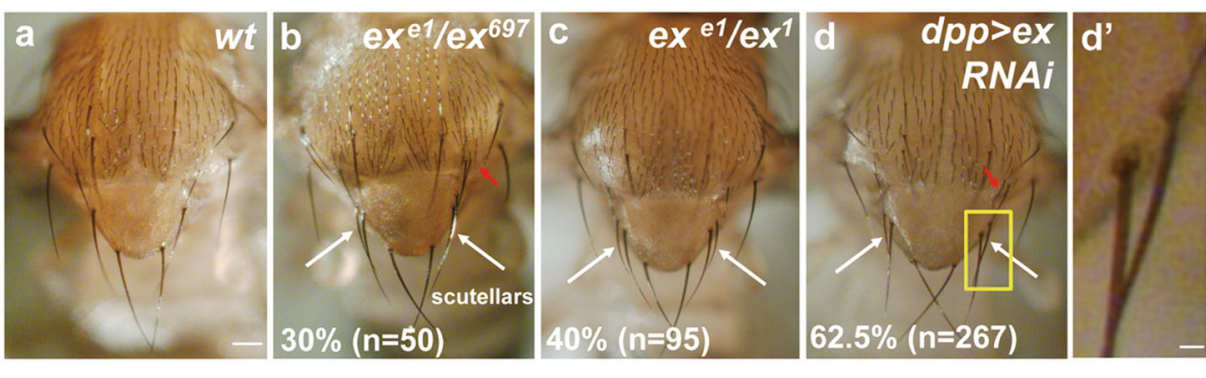

Fig. 1 ex mutations promote generation of extra bristles in Drosophila notum. a Wild-type notal and scutellar bristle pattern. Ectopic bristles develop on the notum of transheterozygous combination of ex mutant alleles (b, c), and ex RNAi driven by dpp-GAL4 flies (d). Note ectopic scutellar (white arrow) and post-alars (red arrow) bristles are observed. $\mathbf{d}^{\prime}$ The enlargement of the boxed area in (d). The numbers indicate the percentages of the population of flies that contained extra macrochaetae in the scutellum. The allelic combinations of ex are used because they are hypomorphic mutants and can survive to adult with minimal growth defects. Scale bars for a-d $100 \mu \mathrm{m} ; \mathbf{d}^{\prime} 10 \mu \mathrm{m}$

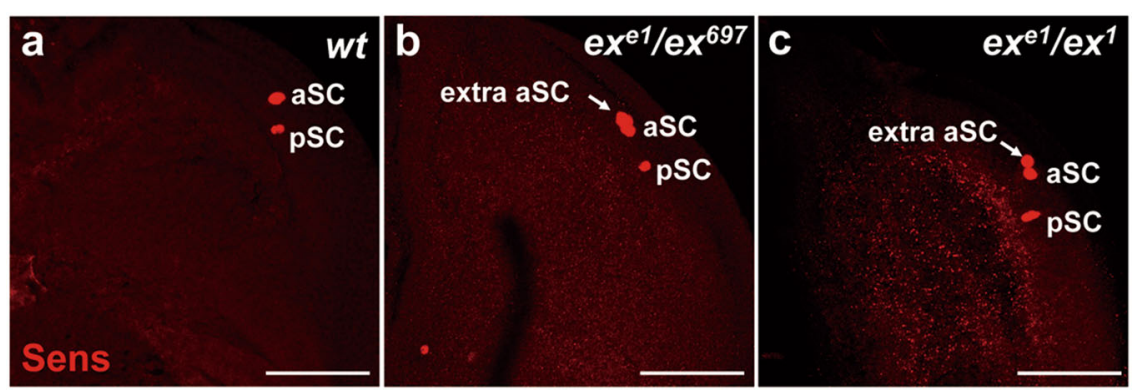

Fig. 2 ex negatively regulates SOP cell formation. a-c The SOP cells in the scutellum of the third-instar larval wing disc are marked by Senseless (Sens, red, a-c). Note that ectopic anterior scutellar (aSC) SOP cells are visualized in allelic combination of ex mutants (b, c). Scale bars, $50 \mu \mathrm{m}$

results indicate the extra macrochaetae of ex mutants are derived from extra SOP cells, not caused by a defect in bristle differentiation or SOP asymmetric division.

\section{Extra macrochaete formation requires Diap1 activity in an ex-dependent manner}

Since Ex functions upstream of the core kinase cassette to regulate SWH pathway activation ${ }^{17}$, mutations of ex led to inhibition of SWH signaling pathway and activation of Yki activity. Consistent with loss-of-function phenotype of ex, ectopic macrochaetae often appeared on the scutellum of flies overexpressing wild-type Yki or the activated $\mathrm{Yki}^{\mathrm{S} 168 \mathrm{~A}}$ (Fig. 3b, c and Supplementary Fig. 2). These observations suggest that Yki activity is sufficient to induce extra bristle formation on scutellum. Moreover, overexpression of a Yki target gene, Diap1, caused extra scutellar bristles under the control of different GAL4 drivers (Fig. 3d, Supplementary Fig. 2 and $^{23}$ ). These data suggest that the SWH pathway is involved in ex-dependent bristle inhibition through modulating Diap1 activity. SWH activity was monitored by using ex intron 3 enhancer (referred to Ex Intron $_{-G F P}$ ) reporter ${ }^{18,19}$, and also

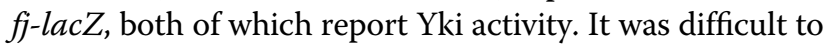
see overall changes in the notum region, although both reporters were present in the ectopic aSC SOP cell (Fig. 4). Collectively, our findings indicate that Yki activation contributes to ectopic macrochaetae formation.

To determine the involvement of Diap1 in the ex mutant phenotype, Diap1 levels were manipulated in ex mutants. As expected, lowered Diap1 levels rescued the extra bristle phenotype of ex mutants while Diap1 transheterozygous mutants have normal patterning and bristle numbers on the notum (Fig. 3f-i). These data indicate that Diap1 is required for extra bristle formation in the absence of ex. The well-characterized function of Diap1 is its role as a caspase inhibitor. To determine the role of caspase activity during bristle determination, caspase activity was blocked by expressing the antiapoptotic proteins baculovirus $\mathrm{p} 35$ or dominant-negative Dronc under the control of $d p p$-GAL4 (Fig. 3e and data not shown). Indeed, blockage of caspase activity led to ectopic bristle formation, which is consistent with previous reports $^{23}$. Since many studies have shown that caspase activation negatively regulates macrochaetae development ${ }^{23,31-33}$, the involvement of Diap1 in bristle formation is likely to be through inhibition of caspase activity. Such caspase-dependent macrochaetae regulation has been shown to represent an apoptosis-independent 


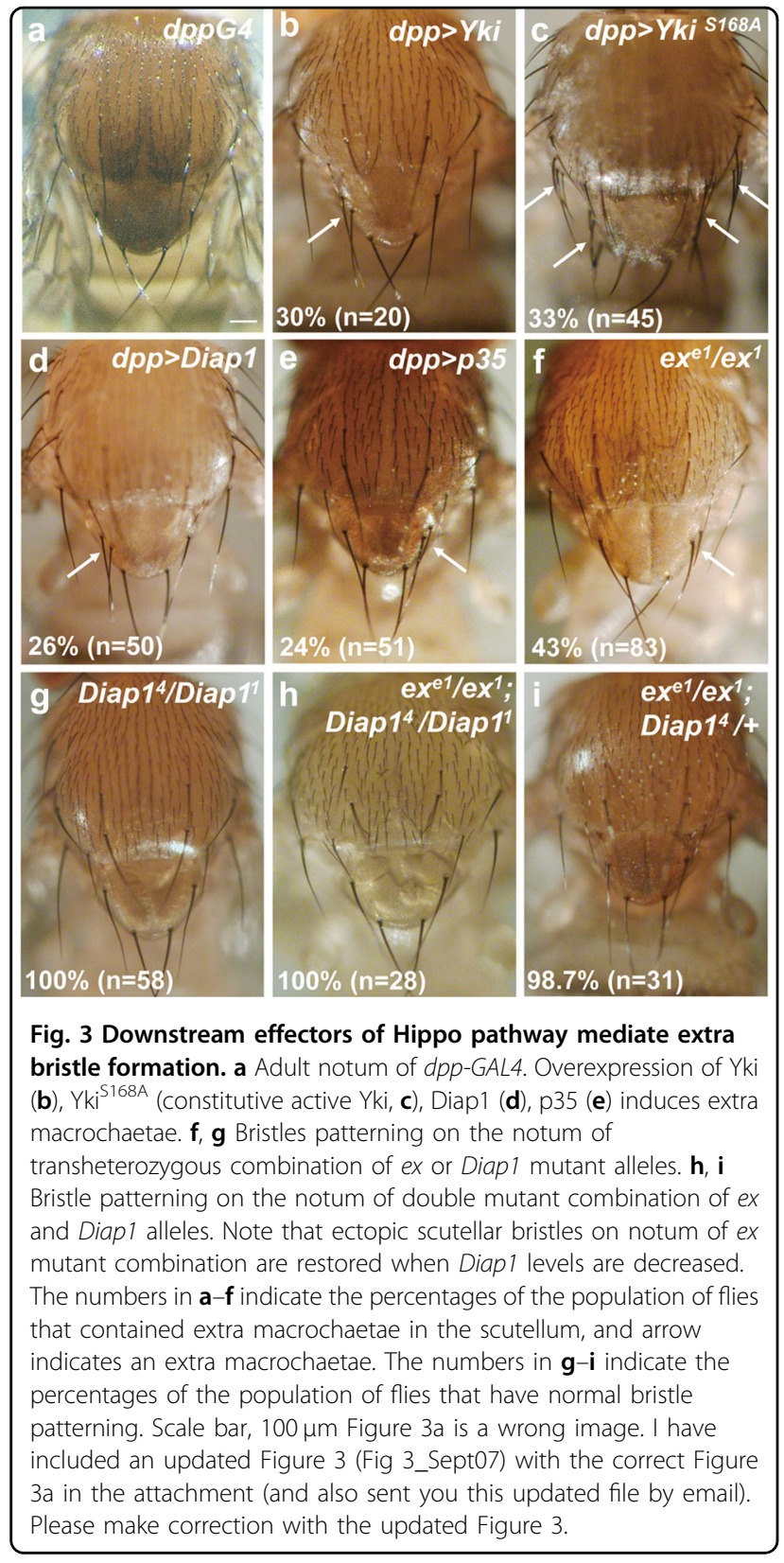

process $^{23,34}$. Like previous authors, we also found no evidence for apoptosis in normal SOP patterning (data not shown).

Wg signaling modulates ex-dependent bristle phenotypes

SOP cells of the macrochaetes arise from proneural clusters in the wing imaginal disc during larval stage. Spatial and temporal patterning of the proneural clusters are established by the expression of proneural genes achaete and scute, which is controlled by multiple cisregulatory elements distributed throughout the achaete and scute transcription units to permit the precision of SOP cell specification ${ }^{35-37}$. Among the critical regulators in modulating proneural gene expression, previous studies have identified Wg/Wnt signaling in particular as affected by nonapoptotic caspase activity during thoracic bristle patterning $^{23}$. Sgg/GSK3 $\beta$ is a negative regulator of $\mathrm{Wg} /$ Wnt signal transduction. ex and $s g g$ mutations shared similar phenotypes in bristle formation (this study; ${ }^{23,38}$ ). Independent evidence also points out that $\mathrm{Wg}$ signaling is altered in the absence of $e x$ in eye discs ${ }^{39}$. Genetic analyses were performed to verify the involvement of $\mathrm{Wg}$ signaling cascade in ex-mediated SOP specification. Indeed, the extra bristle phenotype caused by downregulating ex was suppressed by removing one copy of Wg signaling components (Fig. $5 \mathrm{a}-\mathrm{c}$, e). Similar results were also found when dTcf was knocked down in $d p p>e x$ RNAi flies (Fig. 5d). Overexpression of dominant-negative dTCF (dTCF[DN]) alone prevented macrochaetae formation (yellow bar in Fig. 5f-h). The penetrance of scutellar bristle loss was comparable in ex-knockdown flies with overexpressing dTCF[DN] transgene (magenta bar in Fig. 5f). Although the Notch pathway has been shown to play important role in bristle development ${ }^{40}, \mathrm{E}(\mathrm{spl})$ expression was not changed when ex was mutated (Supplementary Fig. 3), consistent with previous conclusions that ex mutations do not reduce Notch signaling ${ }^{41}$. These results suggest that $\mathrm{Wg}$ signaling pathway acts downstream of $e x$ in SOP cell formation, and therefore that the SWH pathway may by the source of the nonapoptotic caspase activity that acts on Wg signaling.

A caspase-dependent cleavage resulting from caspase activation mediates nonapoptotic signaling in determining SOP cells. This caspase-dependent cleavage is thought to activate Sgg/GSK3 $\beta$ during SOP cell formation ${ }^{23}$. Previous studies have reported that caspasedependent cleavage occurs at the DEVD motif, which has been mapped to DEVD ${ }^{235}$ and $\mathrm{DEVD}^{300}$ of Sgg (Sgg46 isoform) protein ${ }^{23,42}$. Hence, we hypothesize that the caspase inhibitor Diap1 might be the critical effector connecting SWH pathway with Wg pathway through modulating kinase activity of Sgg to determine the correct number of SOP cells. To probe the involvement of Sgg in this regulation in depth, we generated an in vivo noncleavable form of Sgg ( $\left.\mathrm{sg}^{\mathrm{D235G/D300G}}\right)$ using CRISPR-Cas9 technique (thereafter referred to as CRISPR-sgg D235G/D300G, Fig. 6a). The sequence validation of CRISPR-sgg $g^{D 235 G / D 300 G}$ was performed in both genomic DNA and cDNA (Fig. 6b and Supplementary Fig. 4a). If caspase cleavage of $\mathrm{DEVD}^{235}$ or $\mathrm{DEVD}^{300}$ was required to activate Sgg and inhibit Wg signaling during normal bristle development, then $s g g^{D 235 G / D 300 G}$ flies should have elevated Wg signaling and extra bristles, but extra macrochaetae were observed at only a low frequency and only in the first few generations. In case there might be selection for genetic modifiers suppressing the phenotype, we 

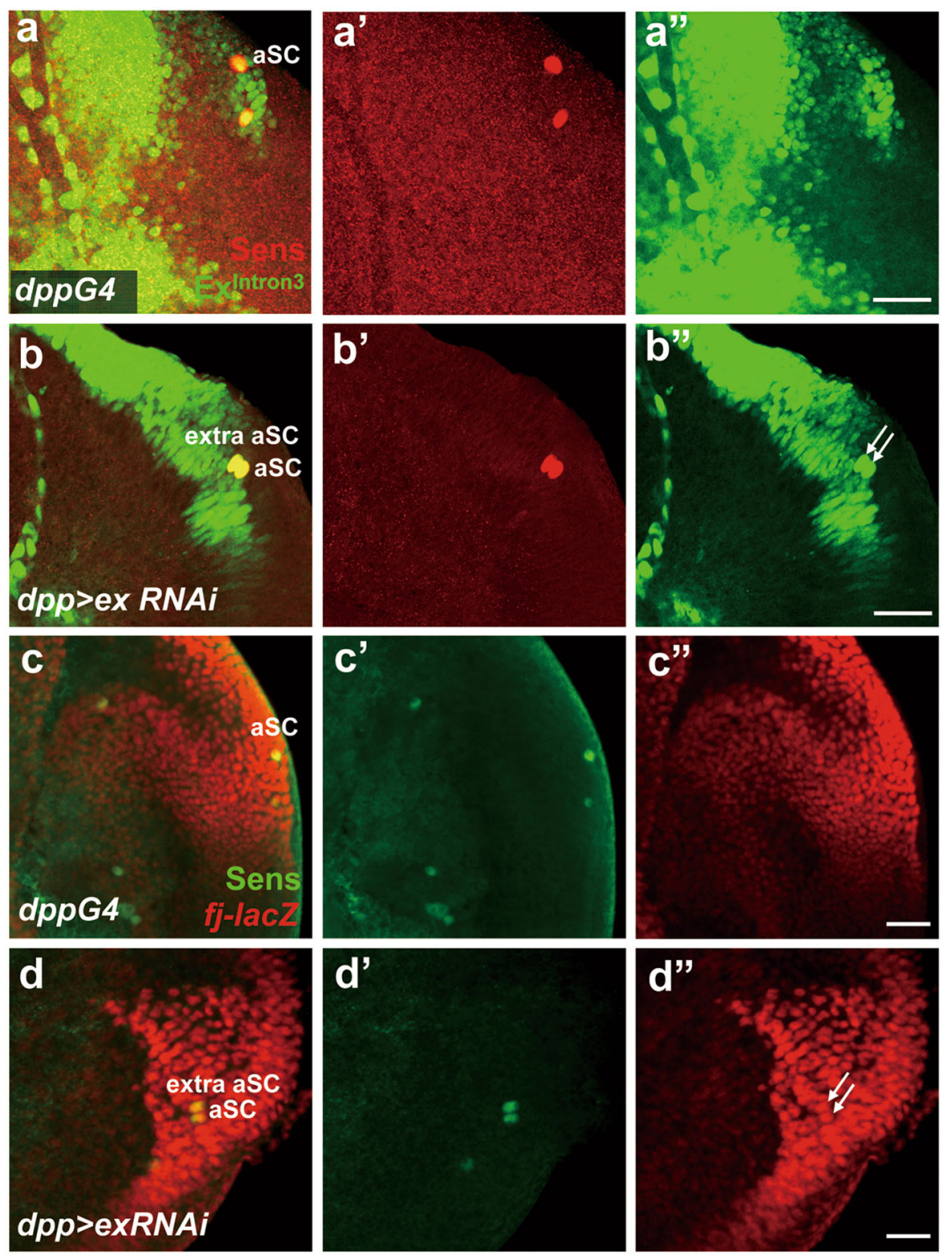

Fig. 4 SWH activity is correlated with ectopic SOP cell. The SOP cells in the scutellum of the third-instar larval wing disc are marked by Sens (red) and Ex Intron3-GFP (green) in dpp-GAL4 (a), and dpp > ex RNAi flies (b). The SOP cells in the scutellum of the third-instar larval wing disc are marked by Sens (green) and fj-lacZ (red) in dpp-GAL4 (c), and dpp > ex RNAi flies (d). White arrow indicates aSC SOP cells (d). Scale bars, $20 \mu \mathrm{m}$

selected $s g g^{D 235 G / D 300 G}$ flies where proximal parts of the $\mathrm{X}$ chromosome were replaced with those from the FRT19 strain after meiotic recombination, and completely exchanged the autosomes by outcrossing at every generation. These flies, which should only be able to retain genetic modifiers on one section of the $\mathrm{X}$ chromosome, exhibited only three extra thoracic macrochaetae in 878 hemizygous $s g g^{D 235 G / D 300 G}$ males (Fig. 6c), a much lower frequency than observed when caspase activity was inhibited (Fig. 3d). Penetrance was similarly low in transheterozygous $\operatorname{sgg}^{1} / C R I S P R$ - sgg ${ }^{D 235 G / D 300 G}$ females (Supplementary Fig. 4b). When ex was knocked down in the CRISPR-sgg ${ }^{D 235 G / D 300 G}$ background, the frequency of extra scutellar macrochaetae was not affected by the $s g g$ mutant background (Supplementary Fig. 4c, d). These observations suggest that Sgg is not the major substrate of caspase-dependent cleavage that affects numbers of macrochaetae in the scutellum. Intriguingly, overexpression of UAS-sgg $g^{D 235 G / D 300 G}$ using Sca-Gal4 resulted in ectopic macrochaete in $19.3 \%$ of flies $^{23}$. This much higher frequency than observed in CRISPR-sgg ${ }^{D 235 G / D 300 G}$ flies suggests that overexpression 

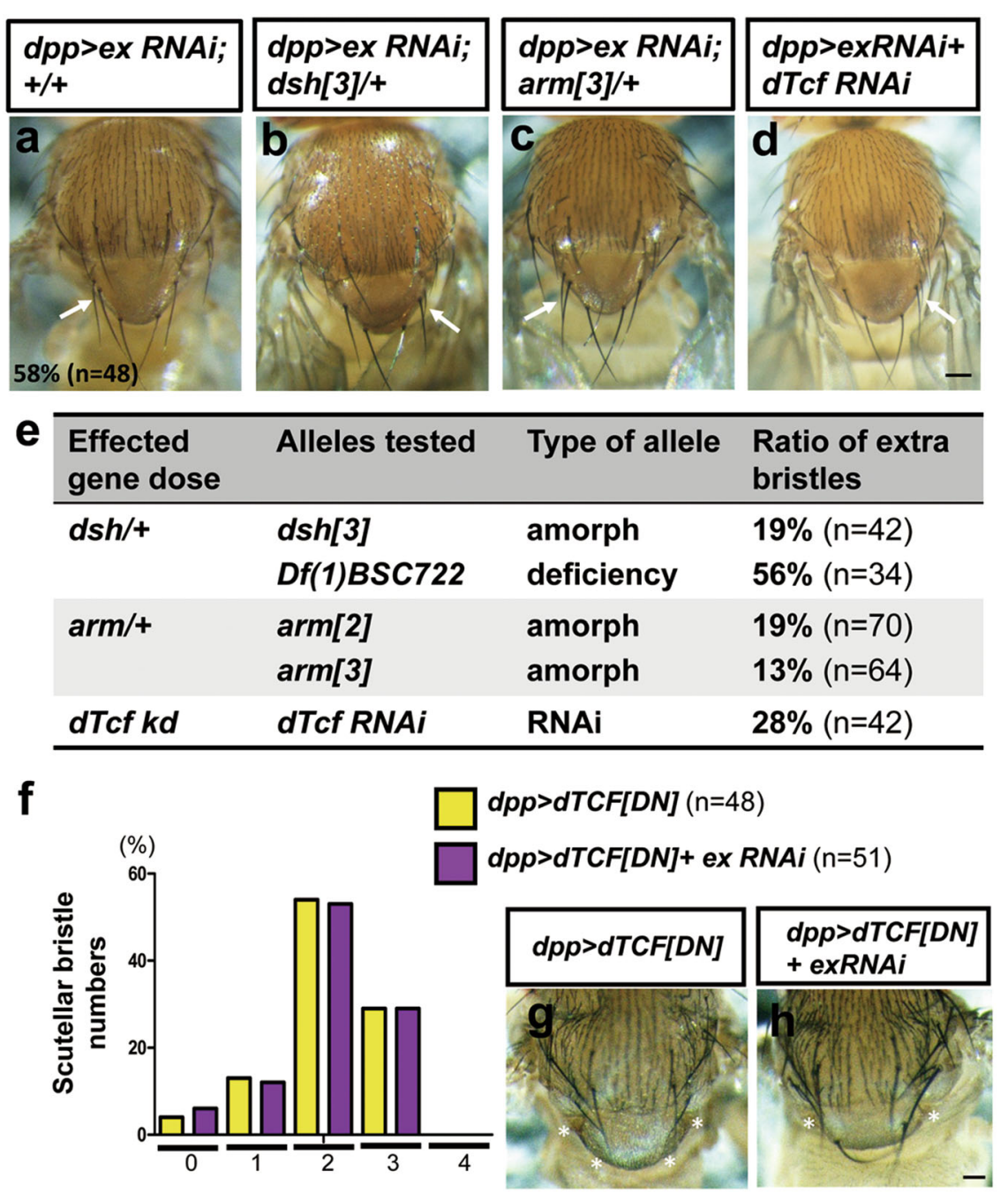

Fig. 5 Genetic interaction between ex and the Wg pathway mutations. a Adult notum of dpp-GAL4, UAS-ex-RNAi. b-d Extra scutellar bristles caused by knockdown of ex are suppressed in decreased levels of Wg pathway components. Genotypes: dsh $h^{3} /+; d p p-G A L 4, U A S-e x-R N A i /+; a r m{ }^{3} /+$; dpp-GAL4,UAS-ex-RNAi/+; w;dpp-GAL4,UAS-ex-RNAi/UAS-dTcf-RNAi. e A table summarized data of b-d and Df(1)BSC722/+;dpp-GAL4,UAS-ex-RNAi/+; $\mathrm{arm}^{2} /+; \mathrm{dpp}-\mathrm{GAL} 4, \mathrm{UAS}-\mathrm{ex}-\mathrm{RNAi} /+$. The numbers indicate the percentages of the population of flies that contained extra macrochaetae. $\mathbf{f} X$ axis represents scutellar bristle numbers $0-4$. $Y$ axis represents the penetrance of indicated scutellar bristle numbers. Wild-type flies normally have four scutellar bristles while overexpression of dTCF[DN] results in a loss of scutellar macrochaetae. Adult nota of w;UAS-dTCF[DN]/+;dpp-GAL4/+ (g) and w;UAS-dTCF[DN]/+;dpp-GAL4,UAS-ex-RNAi/+ (h). Loss of scutellar macrochaetae is indicated by asterisk. Scale bars, $100 \mu \mathrm{m}$

of UAS-sgg D235G/D300G might have dominant negative effects. In sum, our data indicate that there is another target of caspases that affects Wg signaling. Our data cannot rule out some contribution of Sgg cleavage that is redundant with the other target(s).

\section{Discussion}

Unlike the well-known concept that the key roles for SWH pathway are in the regulation of cell proliferation and organ size, the present study reveals a novel function of SWH signaling in cell fate determination through nonapoptotic caspase signaling. As is wellknown, deregulation of SWH pathway leads to the activation of the Yki target gene, Diap1, which restrains caspase activity. Although this can regulate cell survival, here we report that SWH and Yki play a role in normal development in suppressing nonapoptotic caspase activity. In the Drosophila thorax, nonapoptotic caspase activity is needed to suppress activity of Wg signaling and restrain SOP cell specification ${ }^{23}$. This is here shown to depend on the SWH pathway, without which Diap1 expression is too high to permit normal patterning. While this paper was under review, another study reported that nonapoptotic caspase activity is also regulated by the SWH pathway, during tracheal development ${ }^{43}$. 

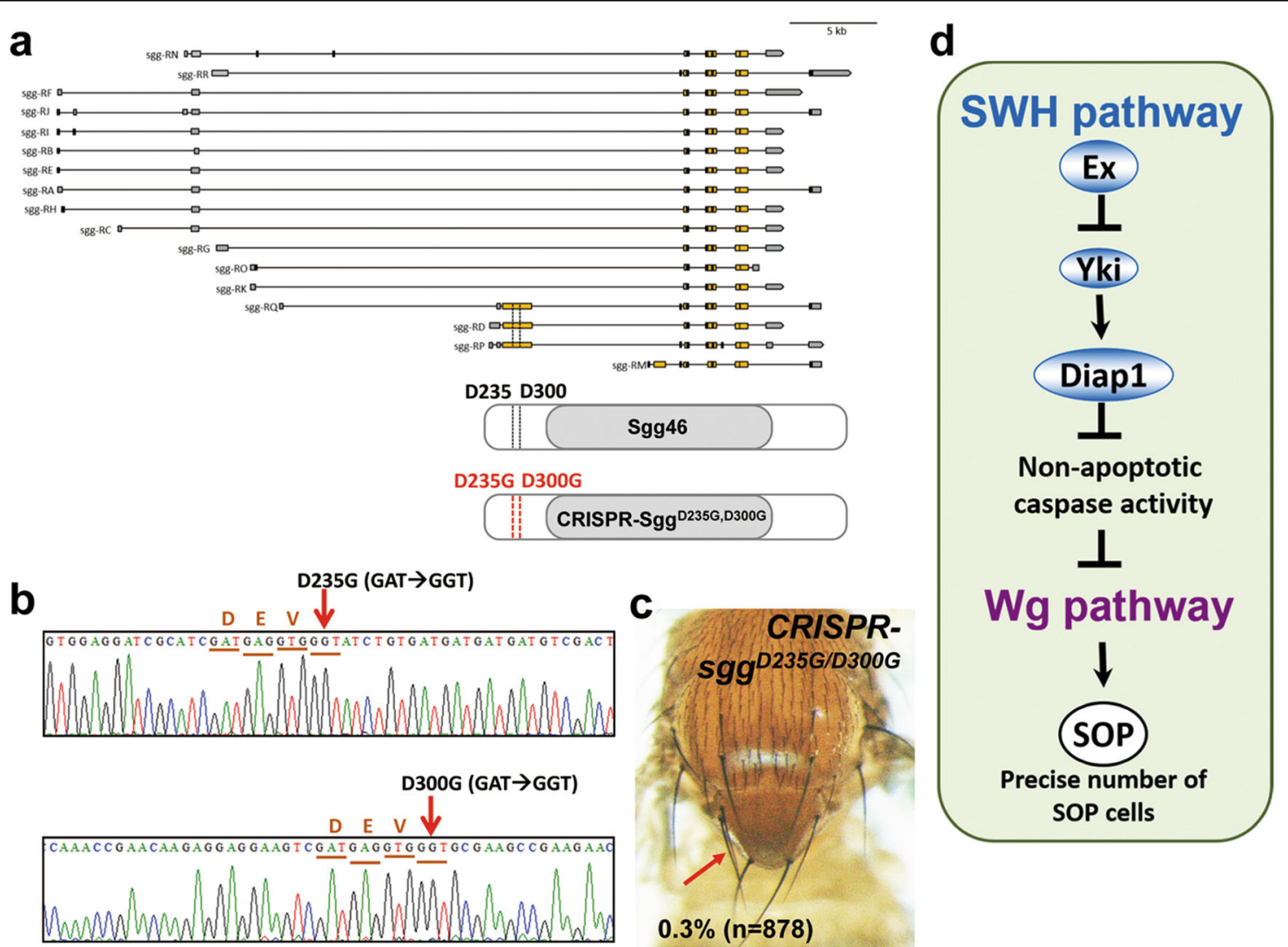

Fig. 6 Sgg is not the only target of nonapoptotic caspase activity. a Schematic representation of the D235 and D300 locus in 3 of 17 sgg isoforms. Exons are represented as yellow boxes, and introns are represented by lines. Noncoding regions are shown as gray boxes. b Sanger sequencing of CRISPR-sgg ${ }^{D 235 G / D 300 G}$ genomic DNA. Note that the corresponding sequences of DEVD235th and DEVD300th residues are mutated from GAT to GGT (glycine). c Bristle patterning of CRISPR-Sgg ${ }^{D 235 G / D 300 G}$ flies. Note the ectopic scutellar bristles (indicated by red arrow) are found in a very low penetrance. $\mathbf{d}$ Model of integrated pathways in regulating SOP specification. Components involving in signal transduction of SWH and Wg pathways are indicated in blue and purple, respectively. The crosstalk between these two pathways is nonapoptotic caspase activity

During SOP specification, caspase activity is transiently controlled by the turnover of Diap1. Diap1 degradation is triggered by the Drosophila IKK-related kinase (DmIKKe)dependent phosphorylation. In consistent with phenotype of high levels of Diap1, downregulation of DmIKKe led to extra macrochaetae formation ${ }^{44}$. Hence, the levels of Diap1 play determinant role in cell fate specification. Here we provide evidence that transcriptional regulation of Diap1 by Yki and the SWH pathway is also important for SOP cell determination, which is disrupted by hypomorphic mutations of ex, which affect the level of Yki activity.

The Drosophila GSK3 $\beta$ ortholog, Sgg, was identified as a potential substrate for caspase-dependent cleavage. One isoform, Sgg46, is inactive but can be cleaved into the active isoform Sgg10, which negatively regulates Wg signaling through the phosphorylation and degradation of $\mathrm{Arm}^{23}$, and also directly phosphorylate Scute and its activator Pannier in SOP cell specification ${ }^{45}$. Caspases potentially have hundreds of substrates, but Sgg46 was believed to be significant for Wg signaling and SOP patterning because overexpression of a form with mutated caspase sites, SggD235G/D300G, phenocopied blockade of nonapoptotic caspases by $\mathrm{p} 35$ overexpression ${ }^{23}$. In fact, SggD235G/D300G overexpression was quantitatively less effective than completely blocking caspases with p35, but this was attributed to the simultaneous presence of wildtype Sgg46 encoded by the endogenous locus ${ }^{23}$. It was presumed that the overexpressed, SggD235G/D300G protein behaved as a competitive inhibitor of Sgg46 cleavage. If this model was correct, we would expect that modifying the endogenous sgg locus to encode $\mathrm{Sgg}^{\mathrm{D} 235 \mathrm{G} / \mathrm{D} 300 \mathrm{G}}$ (which would not affect other, shorter isoforms of Sgg, Fig. 6a), should more completely prevent cleavage of Sgg46 and more completely block the nonapoptotic caspase regulation of $\mathrm{Wg}$ signaling and SOP patterning, resulting in many extra macrochaetae, comparable with ectopic expression of Diap1 or p35. In 
contrast to this expectation, we found almost no phenotypic effect of the endogenous $\operatorname{sgg}^{D 235 G / D 300 G}$ mutant. This is not consistent with the model that Sgg46 is the main target of nonapoptotic caspase signaling in bristle patterning. Although we cannot exclude that Sgg46 might be activated by cleavage at another site, this would not explain why Sgg46 could not be activated when SggD235G/D300G protein was overexpressed. Therefore, we conclude that nonapoptotic caspases regulate one or more other substrates that are critical for Wg signaling and SOP patterning, and that SggD235G/D300G overexpression is a competitive inhibitor of cleavage of these other substrates.

A recent study has reported that the unconventional myosin Crinkled acts as an adapter to facilitate Sgg46 cleavage and activation by Dronc ${ }^{46}$. Aside from our finding that the caspase cleavage sites of Sgg46 are largely dispensable for bristle patterning, the model that Sgg46 is a Dronc target also does not fit with the observation that bristle patterning is disrupted by p35 overexpression $^{23}$, since p35 does not inhibit Dronc. The ectopic p35 phenotype strongly suggests that the major regulators of bristle patterning are substrates of p35dependent effector caspases, not direct Dronc targets. These data could explain how SggD235G/D300G overexpression is dominant negative, however, if SggD235G/ D300G inhibits Dronc and Crinkled function, leading to deficient nonapoptotic signaling by downstream effector caspases.

Our main conclusion is that, in addition to its previously known roles, the SWH pathway is important for regulating nonapoptotic caspase signaling, presumably through Yki control of Diap1 transcription. In the Drosophila thorax this is required to restrain Wg signaling and bristle patterning (Fig. 6d). It is possible that this nonapoptotic caspase signaling might underlie the crosstalk between SWH and Wg signaling in other tissues, such as the eye and wing, the molecular basis of which has so far remained unclear. At one time it was thought that $w g$ was a transcriptional target of Yki but it is now thought this reflects enhanced $w g$ autoregulation when Wg signaling is elevated ${ }^{39,47}$. Crosstalk between SWH and Wnt signaling appears to be conserved, having also been reported in vertebrates ${ }^{48-54}$. The mechanisms that have been suggested in vertebrates are not yet known to involve nonapoptotic caspase signaling, however.

It is striking that in both Drosophila and in mammals, SWH signaling and nonapoptotic caspase signaling both are implicated in neuronal morphogenesis. SWH signaling affects synapse development and dendrite morphogenesis $^{55-59}$, while nonapoptotic roles of caspases remodel Drosophila dendritic arborization neurons and regulate axon degeneration in mammals. Defects of caspase-dependent nonapoptotic signaling affect plasticity and result in disease such as Alzheimer's disease ${ }^{60,61}$. Wnt signaling is also involved in neuronal development and has been associated with neurological diseases including Alzheimer's disease, Parkinson's disease, schizophrenia, and autism ${ }^{62-66}$. Crosstalk between SWH signaling, Wnt signaling and caspase-dependent nonapoptotic signaling may contribute to the molecular mechanisms of neuronal pathogenesis. This crosstalk may occur in multiple processes during development, in light of the finding that DrICE has a nonapoptotic function, which acts downstream of the SWH signaling to regulate endocytic trafficking during tracheal morphogenesis ${ }^{43}$.

\section{Acknowledgements}

We thank Hugo Bellen, Claude Desplan, and Masayuki Miura for fly reagents and Sudershana Nair and Venkateswara Reddy for comments on the paper. We appreciate the information provided by the Flybase ${ }^{67}$. Leica Confocal Imaging was performed in the Analytical Imaging Facility at AECOM supported by the NCI (P30CA013330) and NIH (SIG 1S100D023591-01). Zeiss confocal microscopy was supported by Neuroscience Core Facility of Academia Sinica (AS-CFIl-108-106, Taiwan). L.H.W is funded by the Ministry of Science and Technology of Taiwan (MOST 105-2311-B-016-001-MY2, MOST 107-2311-B-016001-MY3 and MOST 108-3111-Y-016-008). Research in N.E.B's laboratory has been supported by grants from the NIH (GM047892 and EY028990).

\section{Author details}

${ }^{1}$ Graduate Institute of Life Sciences, National Defense Medical Center, $161 \mathrm{Sec}$ 6, Minquan E. Rd, Taipei 11490, Taiwan. ${ }^{2}$ Department of Genetics, Albert Einstein College of Medicine, 1300 Morris Park Avenue, Bronx, NY 10461, USA. ${ }^{3}$ Department of Developmental and Molecular Biology, Albert Einstein College of Medicine, 1300 Morris Park Avenue, Bronx, NY 10461, USA. ${ }^{4}$ Department of Ophthalmology and Visual Sciences, Albert Einstein College of Medicine, 1300 Morris Park Avenue, Bronx, NY 10461, USA

Conflict of interest

The authors declare that they have no conflict of interest.

\section{Publisher's note}

Springer Nature remains neutral with regard to jurisdictional claims in published maps and institutional affiliations.

Supplementary Information accompanies this paper at (https://doi.org/ 10.1038/s41419-019-1924-3).

Received: 2 February 2019 Revised: 3 August 2019 Accepted: 27 August 2019

Published online: 11 September 2019

\footnotetext{
References

1. Yu, F. X., Zhao, B. \& Guan, K. L. Hippo pathway in organ size control, tissue homeostasis, and cancer. Cell 163, 811-828 (2015).

2. Misra, J. R. \& Invine, K. D. The Hippo signaling network and its biological functions. Annu. Rev. Genet. 52, 65-87 (2018).

3. Janse van Rensburg, H. J. \& Yang, X. The roles of the Hippo pathway in cancer metastasis. Cell. Signal. 28, 1761-1772 (2016).

4. Zanconato, F., Cordenonsi, M. \& Piccolo, S. YAP/TAZ at the roots of cancer. Cancer Cell 29, 783-803 (2016).

5. Warren, J. S. A. Xiao, Y. \& Lamar, J. M. YAP/TAZ activation as a target for treating metastatic cancer. Cancers 10, E115 (2018).

6. Pan, D. The Hippo signaling pathway in development and cancer. Dev. Cell 19, 491-505 (2010)

7. Meng, Z., Moroishi, T. \& Guan, K. L. Mechanisms of Hippo pathway regulation. Genes Dev. 30, 1-17 (2016).
} 
8. Huang, J., Wu, S., Barrera, J., Matthews, K. \& Pan, D. The Hippo signaling pathway coordinately regulates cell proliferation and apoptosis by inactivating Yorkie, the Drosophila Homolog of YAP. Cell 122, 421-434 (2005).

9. Zhao, B. et al. Inactivation of YAP oncoprotein by the Hippo pathway is involved in cell contact inhibition and tissue growth control. Genes Dev. 21, 2747-2761 (2007).

10. Oh, H. \& Irvine, K. D. In vivo regulation of Yorkie phosphorylation and localization. Development 135, 1081-1088 (2008).

11. Badouel, C. et al. The FERM-domain protein expanded regulates Hippo pathway activity via direct interactions with the transcriptional activator Yorkie. Dev. Cell 16, 411-420 (2009).

12. Oh, H., Reddy, B. V. \& Irvine, K. D. Phosphorylation-independent repression of Yorkie in Fat-Hippo signaling. Dev. Biol. 335, 188-197 (2009).

13. Ling, C. et al. The apical transmembrane protein Crumbs functions as a tumor suppressor that regulates Hippo signaling by binding to Expanded. Proc. Natl Acad. Sci. USA 107, 10532-10537 (2010).

14. Robinson, B. S., Huang, J., Hong, Y. \& Moberg, K. H. Crumbs regulates Salvador/ Warts/Hippo signaling in Drosophila via the FERM-domain protein expanded. Curr. Biol. 20, 582-590 (2010).

15. Chen, C. L. et al. The apical-basal cell polarity determinant Crumbs regulates Hippo signaling in Drosophila. Proc. Natl Acad. Sci. USA 107, 15810-15815 (2010).

16. Grzeschik, N. A., Parsons, L. M., Allott, M. L., Harvey, K. F. \& Richardson, H. E. Lgl, aPKC, and Crumbs regulate the Salvador/Warts/Hippo pathway through two distinct mechanisms. Curr. Biol. 20, 573-581 (2010).

17. Hamaratoglu, F. et al. The tumour-suppressor genes NF2/Merlin and expanded act through Hippo signalling to regulate cell proliferation and apoptosis. Nat. Cell Biol. 8, 27-36 (2006).

18. Wang, L. H. \& Baker, N. E. Salvador-Warts-Hippo pathway in a developmental checkpoint monitoring helix-loop-helix proteins. Dev. Cell 32 191-202 (2015).

19. Wang, L. H. \& Baker, N. E. Spatial regulation of expanded transcription in the Drosophila wing imaginal disc. PLoS ONE 13, e0201317 (2018).

20. Simpson, P. Lateral inhibition and the development of the sensory bristles of the adult peripheral nervous system of Drosophila. Development 109, 509-519 (1990).

21. Bate C. M. Development of sensory systems in arthropods. in Handbook of Sensory Physiology, Vol. IX (ed Jacobson, M.) 1-53 (Spring-Verlag, Berlin, 1978).

22. Bodmer, R., Carretto, R. \& Jan, Y. N. Neurogenesis of the peripheral nervous system in Drosophila embryos: DNA replication patterns and cell lineages. Neuron 3, 21-32 (1989).

23. Kanuka, $\mathrm{H}$. et al. Drosophila caspase transduces Shaggy/GSK-3beta kinase activity in neural precursor development. EMBO J. 24, 3793-3806 (2005).

24. Boedigheimer, M. \& Laughon, A. Expanded: a gene involved in the control of cell proliferation in imaginal discs. Development 118, 1291-1301 (1993).

25. Cullen, K. \& McCall, K. Role of programmed cell death in patterning the Drosophila antennal arista. Dev. Biol. 275, 82-92 (2004).

26. Hay, B. A., Wassarman, D. A. \& Rubin, G. M. Drosophila homologs of baculovirus inhibitor of apoptosis proteins function to block cell death. Cell 83, 1253-1262 (1995).

27. Perrimon, N. \& Mahowald, A. P. Multiple functions of segment polarity genes in Drosophila. Dev. Biol. 119, 587-600 (1987).

28. Wieschaus, E., Nusslein-Volhard, C. \& Jurgens, G. Mutations affecting the pattern of the larval cuticle in Drosophila melanogaster. Zygotic loci on the Xchromosome and fourth chromosome. Wilhelm. Roux. Arch. Dev. Biol. 193 296-307 (1984).

29. Staehling-Hampton, K., Jackson, P. D., Clark, M. J., Brand, A. H. \& Hoffmann, F. M Specificity of bone morphogenetic protein-related factors: cell fate and gene expression changes in Drosophila embryos induced by decapentaplegic but not 60A. Cell Growth Differ. 5, 585-593 (1994).

30. Baker, N. E., Li, K., Quiquand, M., Ruggiero, R. \& Wang, L. H. Eye development. Methods 68, 252-259 (2014).

31. Kanuka, H. et al. Control of the cell death pathway by Dapaf-1, a Drosophila Apaf-1/CED-4-related caspase activator. Mol. Cell 4, 757-769 (1999).

32. Rodriguez, A. et al. Dark is a Drosophila homologue of Apaf-1/CED-4 and functions in an evolutionarily conserved death pathway. Nat. Cell Biol. 1, 272-279 (1999)

33. Mendes, C. S. et al. Cytochrome c-d regulates developmental apoptosis in the Drosophila retina. EMBO Rep. 7, 933-939 (2006).

34. Nakajima, Y. I. \& Kuranaga, E. Caspase-dependent non-apoptotic processes in development. Cell Death Differ. 24, 1422-1430 (2017).
35. Romani, S., Campuzano, S., Macagno, E. R. \& Modolell, J. Expression of achaete and scute genes in Drosophila imaginal discs and their function in sensory organ development. Genes Dev. 3, 997-1007 (1989).

36. Cubas, P., de Celis, J. F. Campuzano, S. \& Modolell, J. Proneural clusters of achaete-scute expression and the generation of sensory organs in the Drosophila imaginal wing disc. Genes Dev. 5, 996-1008 (1991).

37. Skeath, J. B. \& Carroll, S. B. Regulation of achaete-scute gene expression and sensory organ pattern formation in the Drosophila wing. Genes Dev. 5, 984-995 (1991).

38. Simpson, P. \& Carteret, C. A study of shaggy reveals spatial domains of expression of achaete-scute alleles on the thorax of Drosophila. Development 106, 57-66 (1989).

39. Tyler, D. M. \& Baker, N. E. Expanded and fat regulate growth and differentiation in the Drosophila eye through multiple signaling pathways. Dev. Biol. 305 187-201 (2007)

40. Modolell, J. \& Campuzano, S. The achaete-scute complex as an integrating device. Int. J. Dev. Biol. 42, 275-282 (1998).

41. Maitra, S., Kulikauskas, R. M., Gavilan, H. \& Fehon, R. G. The tumor suppressors Merlin and Expanded function cooperatively to modulate receptor endocytosis and signaling. Curr. Biol. 16, 702-709 (2006).

42. Takemoto, K., Nagai, T., Miyawaki, A. \& Miura, M. Spatio-temporal activation of caspase revealed by indicator that is insensitive to environmental effects. J. Cell Biol. 160, 235-243 (2003).

43. MaSharry, S. S. \& Beitel, G. J. The Caspase-3 homolog DrlCE regulates endocytic trafficking during Drosophila tracheal morphogenesis. Nat. Commun. 10, 1031 (2019).

44. Kuranaga, E. et al. Drosophila IKK-related kinase regulates nonapoptotic function of caspases via degradation of IAPs. Cell 126, 583-596 (2006).

45. Yang, M., Hatton-Ellis, E. \& Simpson, P. The kinase Sgg modulates temporal development of macrochaetes in Drosophila by phosphorylation of Scute and Pannier. Development 139, 325-334 (2012).

46. Orme, M. H. et al. The unconventional myosin CRINKLED and its mammalian orthologue MYO7A regulate caspases in their signalling roles. Nat. Commun. 7, 10972 (2016)

47. Jaiswal, M., Agrawal, N. \& Sinha, P. Fat and Wingless signaling oppositely regulate epithelial cell-cell adhesion and distal wing development in Drosophila. Development 133, 925-935 (2006).

48. Hergovich, A. \& Hemmings, B. A. TAZ-mediated crosstalk between Wnt and Hippo signaling. Dev. Cell 18, 508-509 (2010).

49. Varelas, X. et al. The Hippo pathway regulates Wnt/beta-catenin signaling. Dev. Cell 18, 579-591 (2010).

50. Zecca, M. \& Struhl, G. A feed-forward circuit linking wingless, fat-dachsous signaling, and the warts-hippo pathway to Drosophila wing growth. PLoS Biol. 8, e1000386 (2010).

51. Heallen, T. et al. Hippo pathway inhibits Wnt signaling to restrain cardiomyocyte proliferation and heart size. Science 332, 458-461 (2011).

52. Azzolin, L. et al. Role of TAZ as mediator of Wnt signaling. Cell 151, 1443-1456 (2012).

53. Imajo, M., Miyatake, K., limura, A., Miyamoto, A. \& Nishida, E. A molecular mechanism that links Hippo signalling to the inhibition of Wnt/B-catenin signalling. EMBO J. 31, 1109-1122 (2012)

54. Byun, M. R. et al. Canonical Wnt signalling activates TAZ through PP1A during osteogenic differentiation. Cell Death Differ. 21, 854-863 (2014).

55. Emoto, K., Parrish, J. Z., Jan, L. Y. \& Jan, Y. N. The tumour suppressor Hippo acts with the NDR kinases in dendritic tiling and maintenance. Nature 443, 210-213 (2006).

56. Williams, D. W., Kondo, S., Krzyzanowska, A., Hiromi, Y. \& Truman, J. W. Local caspase activity directs engulfment of dendrites during pruning. Nat. Neurosci. 9. 1234-1236 (2006)

57. Parrish, J. Z., Xu, P. Kim, C. C., Jan, L. Y. \& Jan, Y. N. The microRNA bantam functions in epithelial cells to regulate scaling growth of dendrite arbors in drosophila sensory neurons. Neuron 63, 788-802 (2009).

58. Jiang, N., Soba, P., Parker, E., Kim, C. C. \& Parrish, J. Z. The microRNA bantam regulates a developmental transition in epithelial cells that restricts sensory dendrite growth. Development 141, 2657-2668 (2014).

59. Ultanir, S. K. et al. MST3 kinase phosphorylates TAO1/2 to enable Myosin Va function in promoting spine synapse development. Neuron 84, 968-982 (2014).

60. Mukherjee, A. \& Williams, D. W. More alive than dead: non-apoptotic roles for caspases in neuronal development, plasticity and disease. Cell Death Differ. 24 1411-1421 (2017). 
61. Hollville, E. \& Deshmukh, M. Physiological functions of non-apoptotic caspase activity in the nervous system. Semin. Cell Dev. Biol. 82, 127-136 (2018).

62. Inestrosa, N. C., Montecinos-Oliva, C. \& Fuenzalida, M. Wht signaling: role in Alzheimer disease and schizophrenia. J. Neuroimmune Pharmacol. 7, 788-807 (2012).

63. Ortiz-Matamoros, A., Salcedo-Tello, P. Avila-Munoz, E., Zepeda, A. \& Arias, C. Role of wnt signaling in the control of adult hippocampal functioning in health and disease: therapeutic implications. Curr. Neuropharmacol. 11 465-476 (2013).
64. Hussaini, S. M. et al. Wnt signaling in neuropsychiatric disorders: ties with adult hippocampal neurogenesis and behavior. Neurosci. Biobehav. Rev. 47, 369-383 (2014).

65. Inestrosa, N. C. \& Varela-Nallar, L. Wnt signaling in the nervous system and in Alzheimer's disease. J. Mol. Cell Biol. 6, 64-74 (2014).

66. Purro, S. A., Galli, S. \& Salinas, P. C. Dysfunction of Wnt signaling and synaptic disassembly in neurodegenerative diseases. J. Mol. Cell Biol. 6, 75-80 (2014).

67. Gramates, L. S. et al. FlyBase at 25: looking to the future. Nucleic Acids Res. 45, D663-D671 (2017). 